\title{
Age-associated expression of erythropoietin and its receptor in rat spiral ganglion neurons and its association with neuronal apoptosis and hearing alterations
}

\author{
CHENG ZHONG and XUEYUAN ZHANG \\ Department of Otolaryngology, Southwest Hospital, Third Military Medical University, Chongqing 400038, P.R. China
}

Received October 21, 2015; Accepted November 4, 2016

DOI: $10.3892 / \mathrm{mmr} .2016 .6010$

\begin{abstract}
The present study aimed to determine the expression of erythropoietin (EPO) and the EPO receptor (EPOR) in spiral ganglion neurons (SGNs) in the inner ear of rats of various ages, and the associated neuronal apoptosis and hearing alterations. A total of 15 healthy rats ( $n=30$ ears), were divided into three groups: i) A nominated infant group at post-natal day (PND) 12-14, ii) an adult group at PND 60 and iii) a 3-year postnatal aged group. Auditory brainstem response (ABR) measurements were performed on all rats. EPO and EPOR expression in the inner ear was detected by immunohistochemistry. In situ terminal deoxynucleotidyl transferase dUTP nick end labeling assays were performed to detect the apoptosis of SGNs. The average hearing thresholds of the ABR (decibels above normal hearing level) were 5.625 \pm 4.955 in the infant, $15.000 \pm 8.498$ in the adult and $23.500 \pm 13.134$ in the aged groups. Hearing thresholds for aged and adult rats increased significantly compared with infant rats. However, the difference in latencies of peak I was not significant $(\mathrm{P}>0.05)$. EPO in SGNs was detected during different developmental periods without significant alterations, but were reduced compared with Corti's organ or the stria vascularis. EPOR expression increased significantly from infant to adult stage, and this increased expression was maintained in the aged group. An age-associated increase in the apoptosis of SGNs was detected in all three groups $(\mathrm{P}=0.0347)$. The potential neuroprotective effects of EPO in SGNs may not be revealed during the aging process under natural conditions, and may be associated with spontaneous neuronal apoptosis and consequently, hearing
\end{abstract}

Correspondence to: Dr Xueyuan Zhang, Department of Otolaryngology, Southwest Hospital, Third Military Medical University, 29 Gaotanyan Street, Shapingba, Chongqing 400038, P.R. China

E-mail: swyingsc@hotmail.com

Abbreviations: EPO, erythropoietin; EPOR, erythropoietin receptor; SGNs, spiral ganglion neurons; ABR, auditory brainstem response

Key words: erythropoietin, spiral ganglion neurons, development, apoptosis, hearing diminution. However, the age-associated increase in EPOR in SGNs may exert a role in neuroprotection when necessary, for example in presbycusis.

\section{Introduction}

Sensorineural deafness develops with age and has a severe impact on human health and quality of life (1). The precise underlying mechanisms and etiology of the disease remain to be elucidated, and its prevention and treatment are current areas of research interest in otology (2). Apoptosis or injury of spiral ganglion neurons (SGNs) in the inner ear are considered the primary causes of sensorineural deafness (3). Thus, it is important to investigate potential anti-apoptotic effects on SGNs to identify potential therapeutic targets for the prevention and treatment of sensorineural deafness.

A previous study has suggested that erythropoietin (EPO) exhibits neuroprotective properties via the erythropoietin receptor (EPOR) (4). EPO and its receptor EPOR are the primary factors promoting erythroid progenitor cell proliferation and differentiation in mammals. EPO was previously considered to be a single functional hematopoietic factor. A previous study suggested that EPO is widely distributed throughout the body, in the brain, liver, uterine tissue and kidneys, and demonstrates protective effects on the brain, spinal cord and heart (5).

Furthermore, previous studies have suggested that EPO is expressed in the inner ear of rodents and may play a protective role. Cayé-Thomasen et al (6) first reported the expression of EPO and EPOR in the inner ear of guinea pigs, suggesting that EPO and its receptor may be important in the regulation of SGNs. Berkingali et al (7) revealed that although EPO treatment of cultured SGNs did not improve the survival rate, the neurite length increased significantly, and the role of EPO in SGNs may therefore be primarily associated with neurite extension. Monge Naldi et al (8) demonstrated that EPO had a protective effect on ischemia- and gentamicin-induced hair cell and neuron injury. These studies suggested that the inner ear may be a target for EPO, and that EPO may have a neuroprotective effect on SGNs.

During the progression of sensorineural deafness, the number of apoptotic SGNs increases, despite the presence of EPO. The present study investigated the expression of EPO and EPOR in rat SGNs during development, to determine 
whether their expression alters throughout the aging process and affects their neuroprotective roles. In addition, it was examined how and at what point they exhibit anti-apoptotic effects.

\section{Materials and methods}

Animals. A total of 15 healthy wild-type Sprague Dawley (SD) rats of either sex at different ages were provided by the Third Military Medical University (Chongqing, China) for use in the present study. The experimental animals were divided into three groups: i) An infant group at post-natal day (PND) 12-14 around the onset of hearing, ii) an adult group at PND 60 and iii) an aged group at $\sim 3$-years postnatal. The present study was approved by the Laboratory Animal Welfare and Ethics Committee of the Third Military Medical University. Guidelines from this Ethics Committee to ensure the welfare of laboratory animals were followed in this study, and the welfare of the rodents was optimized to improve their living environments. Rats were maintained under standard laboratory conditions, at $18-27^{\circ} \mathrm{C}$ and $40-70 \%$ humidity under a $12-\mathrm{h}$ light/dark cycle, with free access to clean water and food. All rats were sacrificed by rapid decapitation.

Tissue preparation and immunohistochemistry. All rats were sacrificed by rapid decapitation. Bilateral temporal bones of each rat were dissected to remove the cochleae, and the capsules were fractured to reveal the membranous cochlear duct and the bony modiolus. The modiolus with the encased acoustic ganglion neurons and the attached cochlear duct was dissected and opened in $37^{\circ} \mathrm{C}$ Dulbecco's phosphate-buffered saline. The middle ear was then opened, and the cochlea was carefully perfused with $1.5 \%$ paraformaldehyde. The fixative was slowly injected into the tympanic scale of the basal turn via the round-window plasma membrane. A small opening was made at the cochlear apex to allow cochlear perfusion. The specimens were submersed in the same fixative at $4^{\circ} \mathrm{C}$ to ensure complete fixation of the osseous tissue components. The cochlea was split into two parts by a longitudinal mid-modiolar transaction and was subsequently embedded in paraffin. Sections of 5-10 $\mu \mathrm{m}$ thickness were cut from the embedded tissue and dried at $60^{\circ} \mathrm{C}$ to melt the paraffin.

De-paraffinization of the tissues was performed using xylene, and rehydration was achieved using decreasing concentrations of ethanol. A 10-min Tris-buffered saline rinse was performed, and the endogenous peroxidase activity was blocked by incubation in $3 \% \mathrm{H}_{2} \mathrm{O}_{2}$ for $5 \mathrm{~min}$ followed by a 30-min incubation with the following primary antibodies: Goat anti-EPO (1:50-1:500; N-19; catalog no. sc-1310; Santa Cruz Biotechnology, Inc., Dallas, TX, USA) and rabbit anti-EPOR (1:10-1:500; catalog no. bs-1424R; Bioss Inc. Woburn, MA, USA). Sections were subsequently incubated with anti-goat (1:150; catalog no. SP9000) or anti-rabbit (1:150; catalog no. SP9001) secondary antibodies from immunohistochemistry staining kits purchased from Beijing Zhongshan Golden Bridge Biotechnology Co., Ltd. (Beijing, China). Color was developed with reagents from these kits, according to the manufacturer's protocol. The tissue was rinsed in running tap water for $5 \mathrm{~min}$. Staining was observed under a light microscope.
Apoptosis detection by terminal deoxynucleotidyl transferase-mediated X-dUTP nick end labeling (TUNEL) assay. TUNEL assays were performed on paraffin sections to detect apoptosis, according to the manufacturer's protocol (In Situ Apoptosis Detection kit; Roche Molecular Diagnostics, Pleasanton, CA, USA). The transforming agent peroxidase (POD) was applied so that the staining could be visualized under a light microscope. POD (In Situ Cell Death Detection kit; OriGene Technologies, Inc., Beijing, China) stained positive nuclei brown. The proportion of apoptotic SGNs was calculated by counting 5 randomly selected fields under a microscope. The average proportion represented the ratio of apoptotic neurons to surviving neurons.

Auditory brainstem response (ABR) recording. All animals were used for recording of the ABR, detected in total from 30 ears of 15 rats $(n=5$ at PND 12-14, n=5 at PND 60 and $n=5$ at 3 -year postnatal). The rats were sedated with $400 \mathrm{mg} / \mathrm{kg}$ chloral hydrate (BBI Life Sciences Corporation, Shanghai, China) injected intraperitoneally, in a soundproof chamber. A total of four subcutaneous stainless-steel needle electrodes were positioned at the vertex (positive), left mastoid (negative), right mastoid (negative) and upper/lower lip (ground) of the animal. The resistance between each electrode and the ground electrode was less than $10 \mathrm{k} \Omega$. The ABRs to the probe tone were recorded using a Nicolet Spirit (Natus Medical, Inc., Pleasanton, CA, USA) and stored on a computer for off-line analysis. The recording sampling frequency was $25 \mathrm{kHz}$, and the signal was notch-filtered at $50 \mathrm{~Hz}$, high pass filtered at $0.1 \mathrm{kHz}$ and low pass filtered at $3 \mathrm{kHz}$. The ABR consisted of five vertex-positive peaks, labeled I-V. The latency of peak I exponentially decreased with increasing stimulus intensity. ABR latency was reduced by $2 \mathrm{~ms}$ as the correction for the acoustic travel time from the loudspeakers to the ear. The hearing thresholds, including the amplitude and latency of ABRs, were detected and recorded for analysis.

Statistical analysis. The hearing thresholds and latency of peak I of ABRs for statistical analysis were expressed as the mean \pm standard deviation. Data were analyzed using one-way analysis of variance followed by the least significant difference post hoc test. For immunohistochemistry, five sections from each rat were analyzed by Image-Pro ${ }^{\circledR}$ Plus software version 5.0 (Media Cybernetics, Inc., Rockville, MD, USA), and the average optical densities of positive cells were measured. The percentages of apoptotic SGNs in all groups were analyzed. Data analysis was performed using SPSS software version 13.0 (SPSS, Inc., Chicago, IL, USA). P<0.05 was considered to indicate a statistically significant difference.

\section{Results}

Expression of EPO and EPOR during development. EPO expression was observed in the neurons of the inner ear as brown coarse granulated staining on the plasma membrane and in the cytoplasm surrounding the nuclei. In addition, EPO was present in the organ of Corti, including the hair cells, and in the stria vascularis (Fig. 1A). The expression of EPO in SGNs was detected during different developmental stages without significant alterations (Fig. 1B), and a similar expression 
A
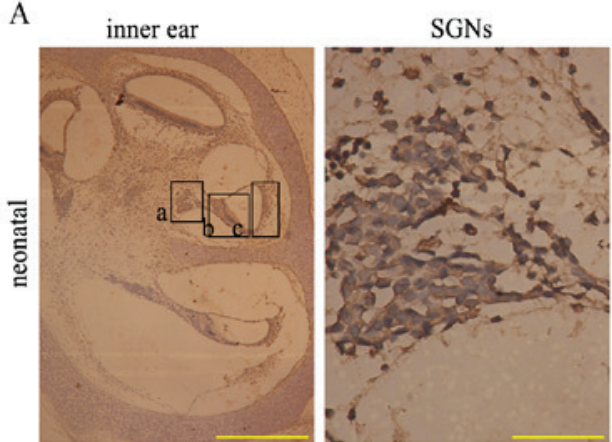

corti's organ

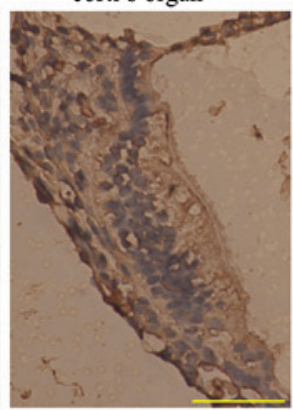

stria vascularis
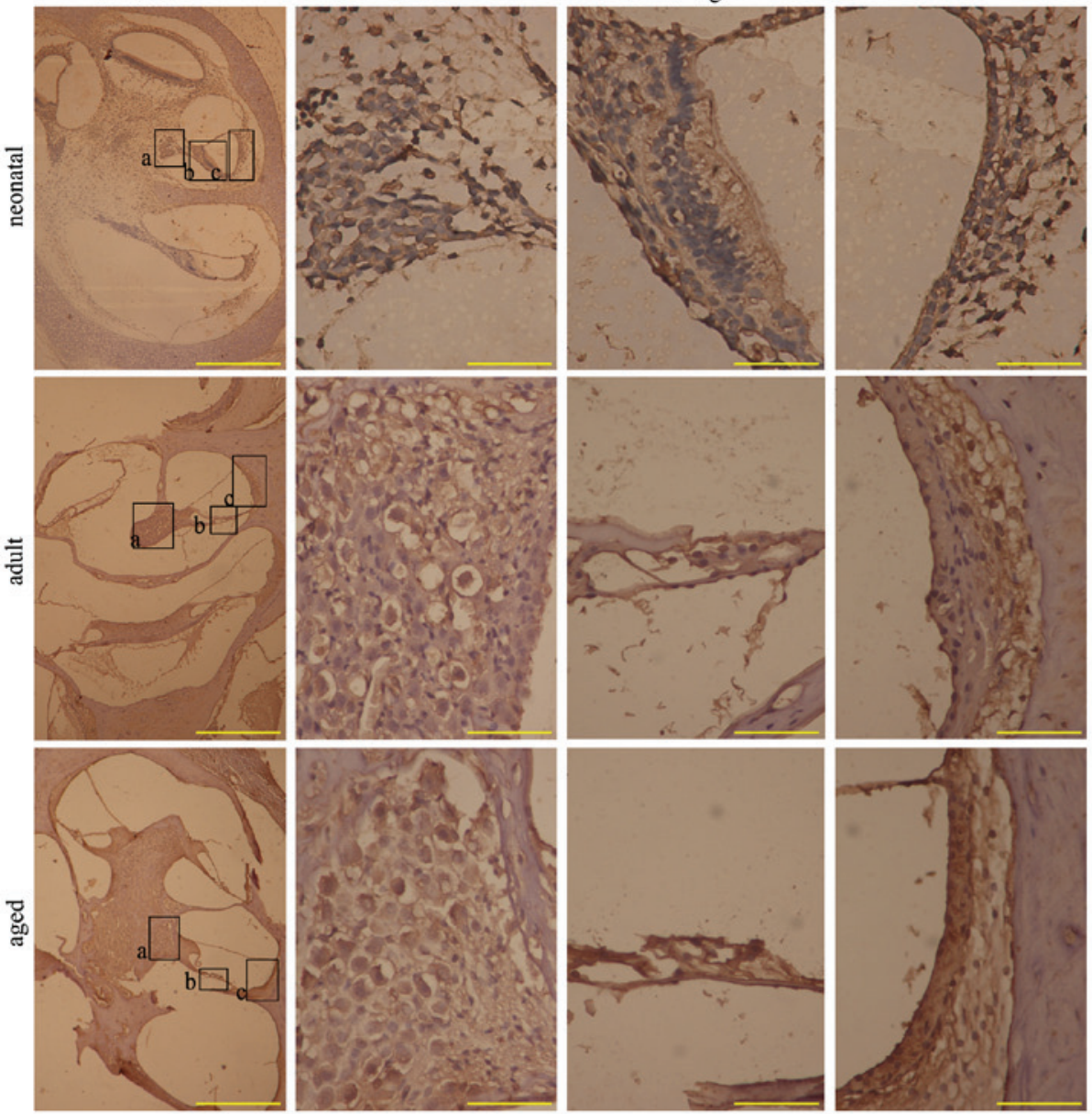

B

Average optical density of EPO in rat inner ear

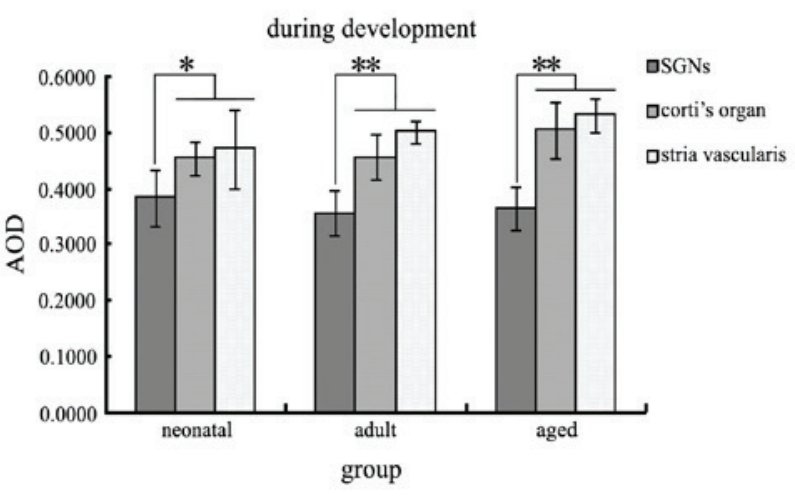

Figure 1. EPO immunohistochemistry in the rat inner ear during development. EPO-positive cells were stained brown primarily in the membrane and cytoplasm. (A) Longitudinal sections of EPO immunohistochemistry in the inner ear, SGNs, Corti's organ and stria vascularis in infant, adult and aged rats. Higher magnification images of the boxed areas a, b and c, in the inner ear images are presented as SGNs, Corti's organ and stria vascularis, respectively. (B) Average optical density of EPO in the rat inner ear during development. The EPO expression levels in the SGNs were significantly reduced compared with the other two areas at all stages. ${ }^{*} \mathrm{P}<0.05$ and ${ }^{* *} \mathrm{P}<0.01$. Inner ear images, scale bar=500 $\mu \mathrm{m}$; all other images, scale bar=50 $\mu \mathrm{m}$. EPO, erythropoietin; SGNs, spiral ganglion neurons; AOD, average optical density.

pattern was observed in Corti's organ and the stria vascularis. However, the EPO expression level in the SGNs was significantly reduced compared with the other two areas $(\mathrm{P}=0.0423$ in infant group; $\mathrm{P}=0.00724$ in adult and aged groups). EPOR expression was observed as clear granulated staining in the plasma membrane and cytoplasm of SGNs in the inner ear, in the phalangeal cells, in the organ of Corti, in the hair cells and in the stria vascularis (Fig. 2A). The expression of EPOR in the SGNs, Corti's organ and stria vascularis increased significantly from the infant to the adult group and this increased expression was maintained in the aged group. There were no significant differences between the adult and aged groups in any of the three areas (Fig. 2B).

Apoptosis of neurons. The nuclei of apoptotic neurons were stained brown (Fig. 3A). The percentage of apoptotic SGNs in 
A
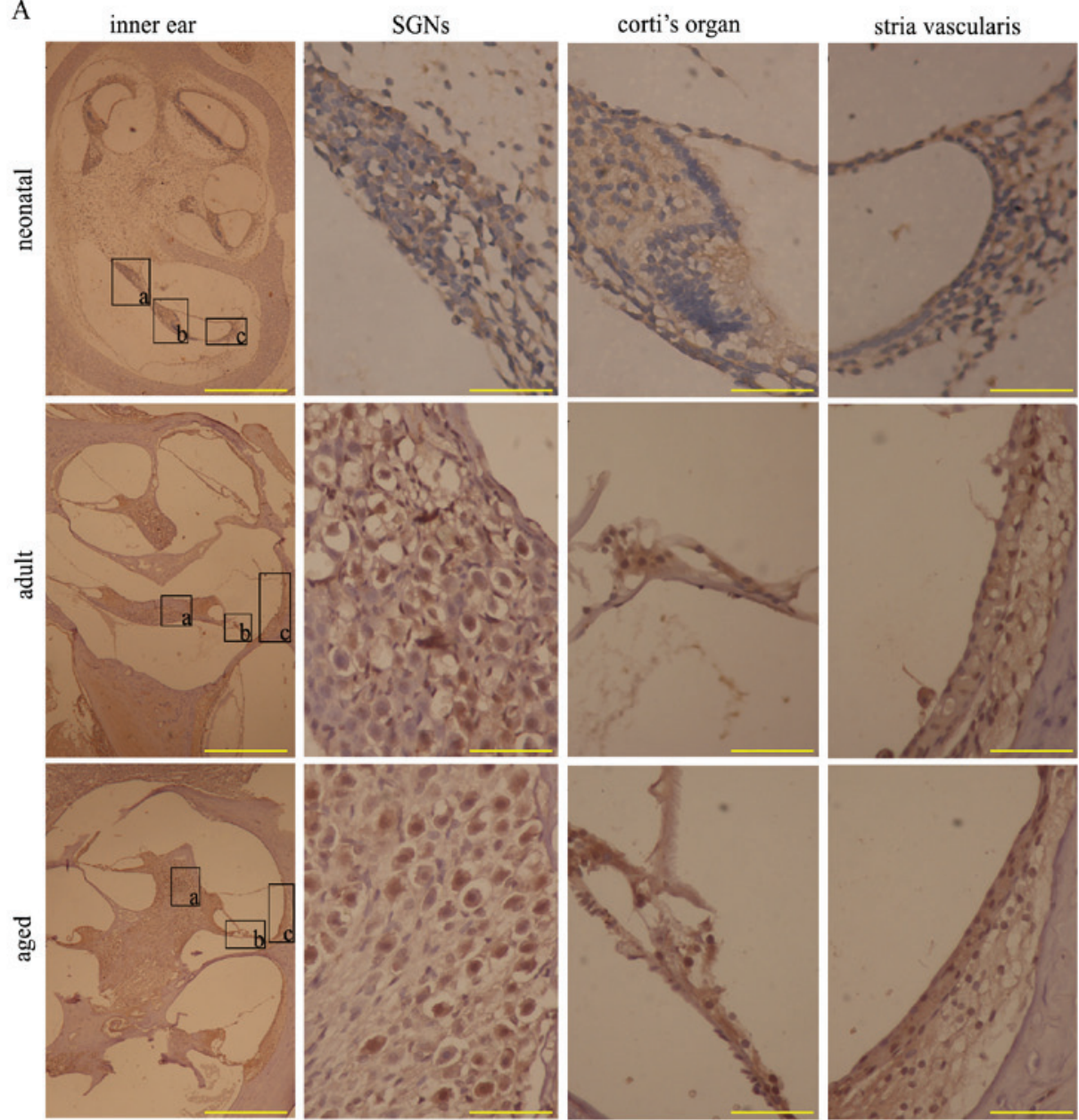

B

Average optical density of EPOR in rat inner ear during development

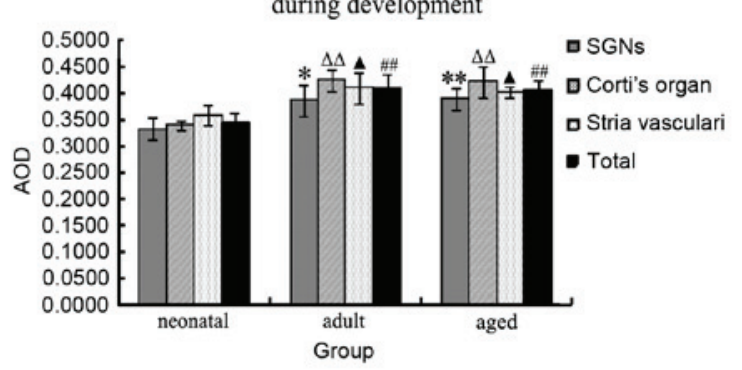

Figure 2. EPOR immunohistochemistry in the rat inner ear during development. EPOR-positive cells were stained brown primarily in the membrane and cytoplasm. (A) Longitudinal sections indicating the EPOR immunohistochemistry reaction in the inner ear, Corti's organ and stria vascularis in infant, adult and aged rats. Higher magnification images of the boxed areas a, b and c, in the inner ear images are presented as SGNs, Corti's organ and stria vascularis, respectively. (B) Average optical density of EPOR in the rat inner ear during development. The expression of EPOR increased significantly from the infant to the adult group and this increased expression was maintained in the aged group. There were no significant differences between the adult and aged groups or in EPOR expression levels in the SGNs, Corti's organ or the stria vascularis. " $\mathrm{P}<0.05$ vs. infant SGNs; ${ }^{* *} \mathrm{P}<0.01$ vs. infant SGNs; ${ }^{\Delta \Delta} \mathrm{P}<0.01$ vs. infant Corti's organ; ${ }^{\triangle} \mathrm{P}<0.05$ vs. infant stria vascularis; ${ }^{\# /} \mathrm{P}<0.01$ vs. infant total. Inner ear images, scale bar=500 $\mu \mathrm{m}$; all other images, scale bar=50 $\mu \mathrm{m}$. EPOR, erythropoietin receptor; SGNs, spiral ganglion neurons; AOD, average optical density.

the infant rat group was $15.11 \pm 0.04 \%$, which was significantly decreased compared with $44.25 \pm 0.11 \%$ in the adult group and $70.23 \pm 0.09 \%$ in the aged group $(\mathrm{P}<0.01$ infant vs. adult/aged groups; Fig. 3B), demonstrating an age-associated increase in neuronal apoptosis.

Audiometric testing of the ABR. The hearing thresholds of rats were recorded in audiograms (Fig. 4A). The average hearing thresholds of the ABR of each ear in the infant, adult and aged groups were $5.625 \pm 4.955,14.500 \pm 8.960$ and $23.500 \pm 13.134$ decibels above normal hearing level, respectively. In the adult rats, the ABR was close to three times that of the infant rats, and in the aged rats, it was close to five times that of the infant rats. Hearing thresholds for adult ( $\mathrm{P}=0.0375$ vs. infant rats) and aged rats $(\mathrm{P}=0.00832$ vs. infant rats) increased significantly (Fig. 4B). Latencies were expressed as the mean \pm standard deviation (infant, $1.796 \pm 0.375 \mathrm{~ms}$; adult, $2.036 \pm 0.093 \mathrm{~ms}$; aged, $1.948 \pm 0.452 \mathrm{~ms}$; Fig. 4C) and amplitudes of peak I in these 
A
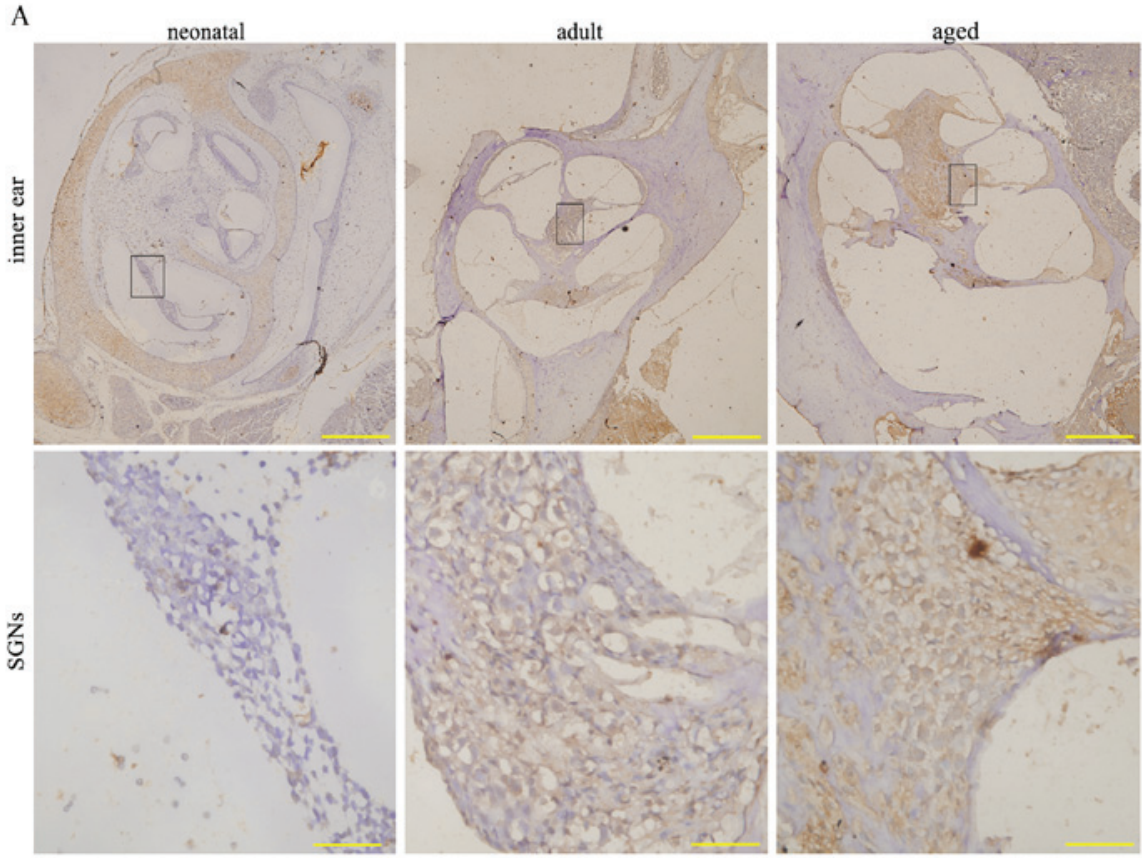

B

Percentage of apoptotic SGNs during development

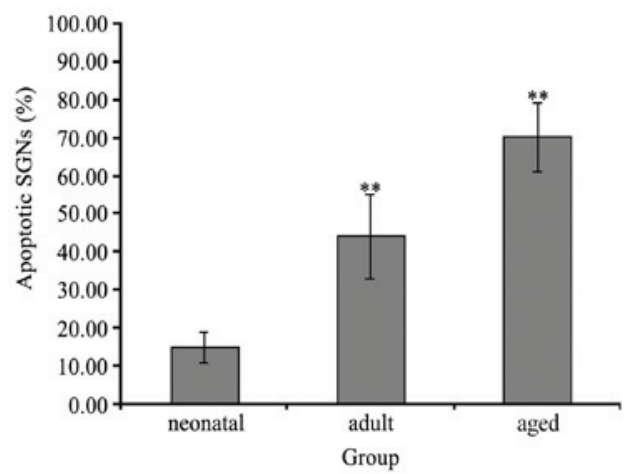

Figure 3. Terminal deoxynucleotidyl transferase-mediated X-dUTP nick end labeling assay revealed apoptotic SGNs during development. Nuclei of apoptotic neurons were stained brown. (A) Apoptotic neurons in the inner ears of infant, adult and aged rats. Higher magnification images of the boxed areas in the top row are presented underneath. (B) The percentage of apoptotic SGNs during development. An age-associated increase in neuronal apoptosis was observed. Top row images, scale bar $=500 \mu \mathrm{m}$; bottom row images, scale bar=50 $\mu \mathrm{m} .{ }^{* *} \mathrm{P}<0.01 \mathrm{vs}$. infant SGNs. SGNs, spiral ganglion neurons.

three groups (infant, 2.036 $\pm 0.905 \mu \mathrm{V}$; adult, 3.089 $\pm 1.308 \mu \mathrm{V}$; aged, $2.181 \pm 1.162 \mu \mathrm{V}$; Fig. 4D) were not significant $(\mathrm{P}=0.079)$.

\section{Discussion}

Sensorineural deafness, the underlying mechanism of which remains to be fully elucidated, is typically characterized by bilateral progressive hearing loss associated with the aging process (9). Injury and apoptosis to the SGNs in the inner ear are considered to be the primary causes of sensorineural deafness (3). SGNs are the primary neurons of the auditory pathway, which transmit signals from auditory hair cells to the auditory center. SGNs are therefore key to successful auditory conduction. The infant rats were assessed at PND 12-14, around the onset of hearing in the present study and compared with mature and aged rats. SGNs in the aged group began to demonstrate a high degree of apoptosis, which was significantly increased compared with the adult group. The hearing of the rats was recorded by ABR in all groups, in a series of five to seven consistent waves that were electrically recorded from the early neural responses to acoustic signals $(10,11)$. The hearing threshold increased significantly with age and was almost 3 and 5 times greater in the adult and aged groups, respectively, compared with the infant group $(1,2)$. Alleviating hearing loss by protecting SGNs has become a primary focus of current research.

The latencies of ABR waves are important otologically and neurophysiologically, and the SGNs (primarily type I), which innervate the inner hair cells, may predominantly contribute to peak I of the ABR (12). Differences in the timing and altitude of the latencies of peak I in the present study were not significantly associated with aging. A previous study has demonstrated that peak latencies are associated with numerous events, from the distance of the recording electrodes to the current source (for example, on the scalp), to local transmembrane postsynaptic currents and the activity of hair cells (13). These alternative explanations may help to explain the findings of the present study.

EPO has demonstrated multiple neuroprotective effects, and has therefore been investigated for its ability to protect 


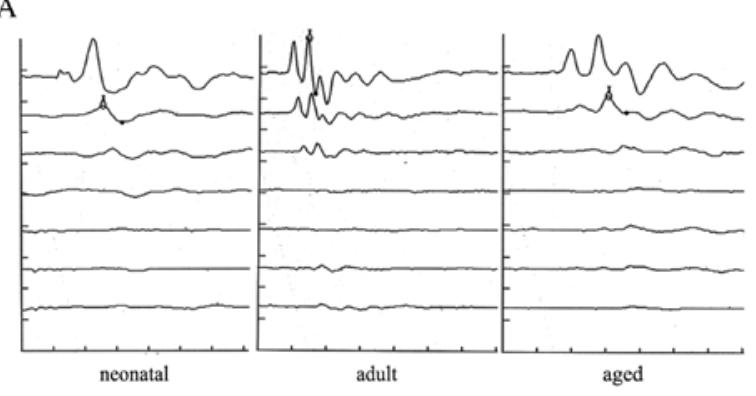

B

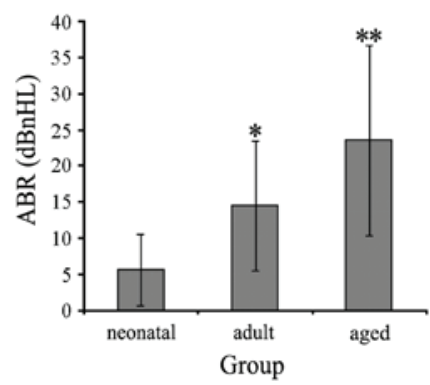

$\mathrm{C}$

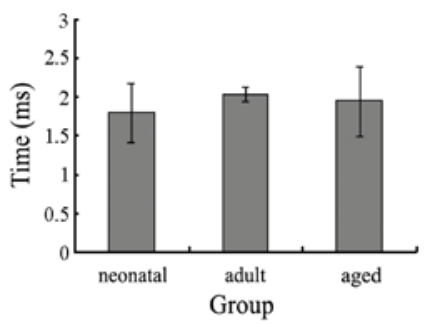

D



Figure 4. ABRs of rats. (A) Audiograms of the infant, adult and aged groups revealed typical peaks of ABR. (B) Average hearing thresholds of infant, adult and old groups were 5.625 $\pm 4.955,15.000 \pm 8.498$ and $23.500 \pm 13.134$ decibels above normal hearing level, respectively. Hearing thresholds of adult and aged rats increased significantly. ${ }^{*} \mathrm{P}<0.05 ;{ }^{* *} \mathrm{P}<0.01$. (C) Average latencies of peak I in the infant, adult and aged groups were $1.796 \pm 0.375,2.036 \pm 0.093$ and $1.948 \pm 0.452 \mathrm{~ms}$, respectively. (D) Average amplitudes of peak I in the infant, adult and aged group were $2.036 \pm 0.905,3.089 \pm 1.308$ and $2.181 \pm 1.162 \mu \mathrm{V}$, respectively. The differences in average time and amplitude of peak I latencies in the three groups were not significant. ABR, audio brainstem response.

SGNs. EPO blocks the translation of apoptosis genes via inhibition of the nuclear translocation of transcription factor $3 \mathrm{a}$, and activates anti-apoptosis genes by promoting the nuclear transfer of nuclear factor (NF)- $\kappa \mathrm{B}(14)$. Sims et al (15) revealed that the cysteine-glutamate transformation ability of neurons differentiating from neural stem cells may be increased by EPO, and thus the neural antioxidation ability may be enhanced. A Previous study demonstrated that the neuroprotective effects of EPO may include at least two mechanisms; a decrease in the hair cell death rate and the induction of angiogenic genes, including the vascular endothelial growth factor and C-X-C chemokine receptor type 4 (16). This data indicates that brain-derived neurotrophic factor and EPO may promote neuronal survival in the inner ear in vitro (17).

Although EPO demonstrates the aforementioned neuroprotective roles and may be detected in SGNs, it does not demonstrate neuroprotective effects on the apoptosis of SGNs during development (thereby ameliorating any sensorineural hearing loss). To address this, the present study detected the expression of EPO in the inner ear, particularly in SGNs during development. It was observed that the expression of EPO did not alter with age in the SGNs, Corti's organ or the stria vascularis. Furthermore, EPO expression in the SGNs was reduced compared with the other two areas, which may be associated with their greater blood flow, and SGNs may be vulnerable and refractory as this area is not highly vascularized.

Previous studies have suggested that circulating EPO, produced primarily by the kidneys, may not cross the blood-brain barrier (BBB), and thus only exerts its effects on neurons in an autocrine or paracrine, but not endocrine, manner $(18,19)$. However, a separate study has revealed that EPO may cross the BBB (20). The antibodies used in the present study did not differentiate between circulating EPO and EPO produced in the inner ear; however, it is possible that EPO expression levels in Corti's organ and the stria vascularis were greater compared with SGNs due to their containing two types of EPO, that is, the circulating EPO and the EPO expressed by the inner ear itself, whereas the SGNs contained reduced levels of circulating EPO.

Frederiksen et al (21) reported contradictory effects of EPO compared with those that had been previously reported. This study observed that EPO augmented noise-induced hearing loss by altering the dynamics of blood flow to the cochlear vascular bed. The authors suggested an underlying mechanism of potentially induced vasoconstriction, which included pathophysiologic alterations and reduced cochlear blood flow, including localized periods of stasis, vascular permeability alterations and local ischemia. These alterations may result in temporary or even permanent deafness. The discrepancies between the findings of this study and others may be correlated with the results of the present study that the expression of EPO and EPOR in the stria vascularis was greater compared with the SGNs. Thus, further research is necessary to investigate whether the expression patterns of EPO in the inner ear impact its role in SGNs.

During development, the expression of EPO in SGNs remained low and did not increase with age, whereas the number of apoptotic SGNs increased with age. Thus, the neuroprotective effects of naturally expressed EPO in SGNs may not be sufficient to counter SGN apoptosis, particularly in aging, as the proposed neuroprotective effects of EPO did not appear in the normal aging process.

The neuroprotective effects of EPO may depend upon the EPO-EPOR interaction. The EPO interaction with EPOR may 
subsequently activate multiple downstream signaling pathways, including Ras/mitogen activated protein kinase, phosphoinositide 3-kinase/protein kinase B, signal transducer and activator of transcription 5 and $\mathrm{NF}-\kappa \mathrm{B}$, resulting in a series of anti-apoptotic priming mechanisms, which exert neuroprotective effects (22). Although in the present study the expression of EPO in the inner ear, especially in the SGNs, indicated no significant alterations during development, the expression of EPOR was significantly increased from infant to adult rats, and this increased expression was maintained in old age. The significance of this alteration in expression is unknown. As neurotrophic factors promote neural differentiation and survival via the aforementioned signaling pathways, this increased expression of EPOR may be associated with the expression of neurotrophic factors; this requires further study. Although EPO expression does not increase in the inner ear, particularly in SGNs, during development, the increased EPOR expression level does exhibit a further neuroprotective role by rendering a high probability of abundant binding anchors for exogenous EPO.

In conclusion, the present study detected the hearing threshold, apoptosis rate and expression of EPO and EPOR in SGNs in infant, adult and aged rats using ABR, TUNEL assay and immunohistochemistry, respectively. The present study demonstrated that the number of apoptotic SGNs and the hearing threshold increased significantly with age. However, the expression of EPO did not alter significantly with age and was maintained at a relatively low level in the SGNs. The present study hypothesized that the neuroprotective effects of EPO expressed in SGNs may not be sufficient to counter the apoptosis of SGNs, particularly in old age. Thus, the neuroprotective effects of EPO were not revealed in the aging process under natural conditions. The expression of EPOR increased significantly from the infant to adult period, and this increased expression was maintained at a high level from the adult to the aged period, which may act as a potential novel therapeutic strategy against age-associated sensorineural deafness via administration of an exogenous supply of EPO.

\section{Acknowledgements}

The present study was supported by the National Natural Science Foundation of China (grant no. 81100720).

\section{References}

1. Monini S, Filippi C, Baldini R and Barbara M: Perceived disability from hearing and voice changes in the elderly. Geriatr Gerontol Int 15: 147-155, 2015.

2. Yamasoba T,Lin FR,Someya S,Kashio A, Sakamoto T and Kondo K: Current concepts in age-related hearing loss: Epidemiology and mechanistic pathways. Hear Res 303: 30-38, 2013.
3. Bao J and Ohlemiller KK: Age-related loss of spiral ganglion neurons. Hear Res 264: 93-97, 2010.

4. Byts N and Sirén AL: Erythropoietin: A multimodal neuroprotective agent. Exp Transl Stroke Med 1: 4, 2009.

5. Brines $M$ and Cerami A: Discovering erythropoietin's extra-hematopoietic functions: Biology and clinical promise. Kidney Int 70: 246-250, 2006.

6. Cayé-Thomasen P, Wagner N, Lidegaard Frederiksen B, Asal K and Thomsen J: Erythropoietin and erythropoietin receptor expression in the guinea pig inner ear. Hear Res 203: 21-27, 2005.

7. Berkingali N, Warnecke A, Gomes P, Paasche G, Tack J, Lenarz T and Stöver T: Neurite outgrowth on cultured spiral ganglion neurons induced by erythropoietin. Hear Res 243: 121-126, 2008.

8. Monge Naldi A, Gassmann M and Bodmer D: Erythropoietin but not VEGF has a protective effect on auditory hair cells in the inner ear. Cell Mol Life Sci 66: 3595-3599, 2009.

9. Panza F, Solfrizzi V and Logroscino G: Age-related hearing impairment-a risk factor and frailty marker for dementia and AD. Nat Rev Neurol 11: 166-175, 2015.

10. Jewett DL and Williston JS: Auditory-evoked far fields averaged from the scalp of humans. Brain 94: 681-696, 1971.

11. Malherbe TK, Hanekom T and Hanekom JJ: Can subject-specific single-fibreelectrically evoked auditory brainstem response data be predicted from a model? Med Eng Phys 35: 926-936, 2013.

12. Rattay F and Danner SM: Peak I of the human auditory brainstem response results from the somatic regions of type I spiral ganglion cells: Evidence from computer modeling. Hear Res 315: 67-79, 2014

13. Brown DJ and Patuzzi RB: Evidence that the compound action potential (CAP) from the auditory nerve is a stationary potential generated across dura mater. Hear Res 267: 12-26, 2010.

14. Chong ZZ and Maiese K: Erythropoietin involves the phosphatidylinositol 3-kinase pathway, 14-4-4 protein and FOXO3a nuclear traffic-king to preserve endothelia cell integrity. Br J Pharmacol 150: 839-850, 2007.

15. Sims B, Clarke M, Njah W, Hopkins ES and Sontheimer H: Erythropoietin-induced neuroprotection requires cystine glutamate exchanger activity. Brain Res 1321: 88-95, 2010.

16. Gross J, Moller R, Amarjargal N, Machulik A, Fuchs J, Ungethüm U, Kuban RJ, Henke W, Haupt H and Mazurek B: Expression of erythropoietin and angiogenic growth factors following inner ear injury of newborn rats. Prague Med Rep 110: 310-331, 2009.

17. Kaiser O, Paasche G, Stöver T, Ernst S, Lenarz T, Kral A and Warnecke A: TGF-beta superfamily member activin A acts with $\mathrm{BDNF}$ and erythropoietin to improve survival of spiral ganglion neurons in vitro. Neuropharma 75: 416-425, 2013.

18. Juul SE, Stallings SA and Christensen RD: Erythropoieti n in the cerebrospinal fluid of neonates who sustained CNS injury. Pediatr Res 46: 543-547, 1999.

19. Saunders NR, Habgood MD and Dziegielewska KM: Barrier mechanisms in the brain, II. Immature brain. Clin Exp Pharmacol Physiol 26: 85-91, 1999.

20. Banks WA, Jumbe NL, Farrell CL, Niehoff ML and Heatherington AC: Passage of erythropoietic agents across the blood-brain barrier: A comparison of human and murine erythropoietin and the analog darbepoetin alfa. Eur J Pharmacol 505: 93-101, 2004.

21. Frederiksen BL, Cayé-Thomasen P, Lund SP, Wagner N, Asal K, Olsen NV and Thomsen J: Does erythropoietin augment noise induced hearing loss? Hear Res 223: 129-137, 2007.

22. Maiese K, Chong ZZ, Li F and Shang YC: Erythropoietin: Elucidating new cellular targets that broaden therapeutic strategies, Prog Neurobiol 85: 194-213, 2008. 